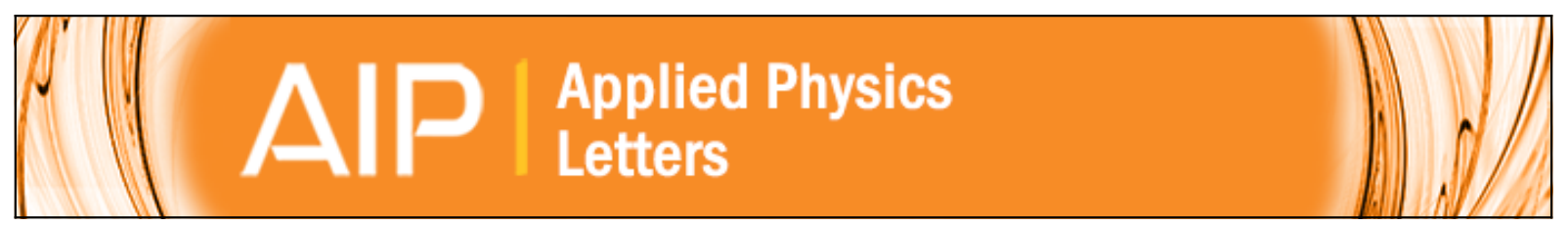

\title{
Reversed rainbow with a nonlocal metamaterial
}

Tiago A. Morgado, João S. Marcos, João T. Costa, Jorge R. Costa, Carlos A. Fernandes, and Mário G.

Silveirinha

Citation: Applied Physics Letters 105, 264101 (2014); doi: 10.1063/1.4905152

View online: http://dx.doi.org/10.1063/1.4905152

View Table of Contents: http://scitation.aip.org/content/aip/journal/apl/105/26?ver=pdfcov

Published by the AIP Publishing

\section{Articles you may be interested in}

Planar metamaterial-based beam-scanning broadband microwave antenna

J. Appl. Phys. 115, 194901 (2014); 10.1063/1.4876233

Measuring trapped noise in metamaterials

J. Appl. Phys. 115, 084905 (2014); 10.1063/1.4866360

Electron beam coupling to a metamaterial structure

Phys. Plasmas 20, 083116 (2013); 10.1063/1.4817021

Design, fabrication, and measurement of highly sub-wavelength double negative metamaterials at high frequencies

J. Appl. Phys. 113, 213712 (2013); 10.1063/1.4809769

Broadband and low loss high refractive index metamaterials in the microwave regime Appl. Phys. Lett. 102, 091108 (2013); 10.1063/1.4794088

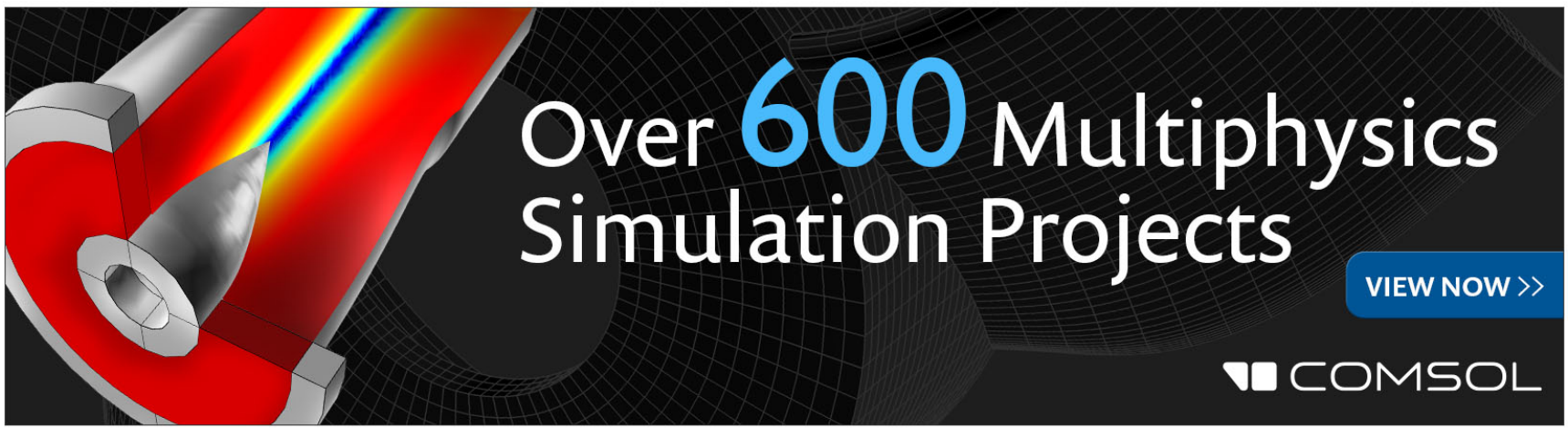




\title{
Reversed rainbow with a nonlocal metamaterial
}

\author{
Tiago A. Morgado, ${ }^{1, a)}$ João S. Marcos, ${ }^{1}$ João T. Costa, ${ }^{2}$ Jorge R. Costa, ${ }^{3}$ \\ Carlos A. Fernandes, ${ }^{4}$ and Mário G. Silveirinha ${ }^{1, b)}$ \\ ${ }^{1}$ Department of Electrical Engineering, Instituto de Telecomunicações, University of Coimbra, 3030 Coimbra, \\ Portugal \\ ${ }^{2}$ CST AG, Bad Nauheimer Strasse 19, 64289 Darmstadt, Germany \\ ${ }^{3}$ Instituto de Telecomunicações and Instituto Universitário de Lisboa (ISCTE-IUL), 1649-026 Lisboa, Portugal \\ ${ }^{4}$ Instituto de Telecomunicações, and Instituto Superior Técnico, Universidade de Lisboa, 1049-001 Lisboa, \\ Portugal
}

(Received 14 November 2014; accepted 16 December 2014; published online 29 December 2014)

\begin{abstract}
One of the intriguing potentials of metamaterials is the possibility to realize a nonlocal electromagnetic reaction, such that the effective medium response at a given point is fundamentally entangled with the macroscopic field distribution at long distances. Here, it is experimentally and numerically verified that a microwave nonlocal metamaterial formed by crossed metallic wires enables a low-loss broadband anomalous material response such that the refractive index decreases with frequency. Notably, it is shown that an electromagnetic beam refracted by our metamaterial prism creates a reversed microwave rainbow. (C) 2014 AIP Publishing LLC.
\end{abstract}

[http://dx.doi.org/10.1063/1.4905152]

Rainbows are among the most stunning optical effects in nature and have captured the human imagination for centuries. Such a ubiquitous phenomenon is usually observed when light passes through a glass prism or water droplets, and is a consequence of the frequency dispersion inherent to the materials. ${ }^{1}$ Causality and passivity constraints ${ }^{2}$ impose that the refractive index of a conventional low-loss dielectric material is a strictly increasing function of frequency. ${ }^{2}$ Thus, colors associated with longer wavelengths are less refracted than colors associated with shorter wavelengths, originating the usual rainbow patterns.

Metamaterials with tailored electromagnetic responses continue to be one of the most vibrant fields of research in applied physics because of their transformational properties. $^{3-8}$ An intriguing potential of metamaterials is the possibility to realize a nonlocal response. In a nonlocal material, the polarization vector at a given point of space does not depend exclusively on the macroscopic electric field in a small neighborhood of that point: it may depend on the electric field distribution in the whole crystal. Most of the conventional (solid-state) materials behave, to a good approximation, as local media. The electrodynamics of nonlocal materials is much richer than in conventional media, and this can have applications in the transport and manipulation of the near-field, ${ }^{9-14}$ ultra-subwavelength waveguiding, ${ }^{15-17}$ near-field superlensing, ${ }^{18,19}$ and broadband allangle negative refraction, ${ }^{20,21}$ amongst others.

To have a strong non-locality, the metamaterial topology needs to be fundamentally different from those characteristic of natural crystalline materials, wherein the atoms lie isolated at the lattice points (cermet topology) and nearest neighbor coupling dominates. The trick is to interconnect all the structural unities of the metamaterial (network topology)

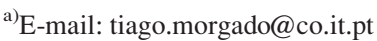

b) Author to whom correspondence should be addressed. Electronic mail: mario.silveirinha@co.it.pt
}

such that the response of each individual meta-atom becomes fundamentally entangled with those of other meta-atoms located at large distances, giving rise to a strong nonlocal response. As shown in previous works, this can be achieved based on crossed metallic wire inclusions that span several unit cells of the material ("double" wire medium"15,22,23,25). With such a solution, the polarization acquired by each meta-atom (formed by segments of the metallic wires) strongly depends on the macroscopic electric field in far distant cells, because the electric current flowing along the wires must be continuous across cells. In general, metamaterials formed by periodic arrays of metallic wires ${ }^{24-26}$ are characterized by a strong spatial dispersive electromagnetic response so that the dielectric function depends both on the oscillation frequency $\omega$ and the wave vector $\mathbf{k}$ (i.e., $\varepsilon=\varepsilon(\omega, \mathbf{k})){ }^{24}$ Wire media can have relevant applications in the context of negative refraction, ${ }^{27}$ far-field superlensing, ${ }^{28}$ enhancement of the Vavilov-Cherenkov emission, 29,30 enhancement of spontaneous emission and light-matter interactions, ${ }^{31,32}$ radiative heat transport, ${ }^{33}$ and biosensing. ${ }^{34}$

As theoretically demonstrated in Ref. 35, one of the exciting opportunities created by the strong nonlocal response of the double-wire array is the realization of media with a low-loss broadband anomalous material dispersion, such that the refractive index decreases with frequency (Fig. 1), i.e., $d n / d \omega<0$ ( $n$ represents the refractive index of the material). ${ }^{35}$ Even though regimes of anomalous dispersion are also possible with conventional dielectrics, ${ }^{36-38}$ the physical origin and nature of these effects are rather distinct from the proposal of Ref. 35. An anomalous dispersion in usual dielectric materials is narrowband and creates a very lossy effect due to absorption resonances. ${ }^{36,37}$ Quite differently, the anomalous material dispersion reported in Ref. 35 is a low-loss broadband property rooted in the specific topology of the material. It may be attributed to the fact that long metallic wires tend to obstruct the wave propagation more effectively for low frequencies, because the wires length is 


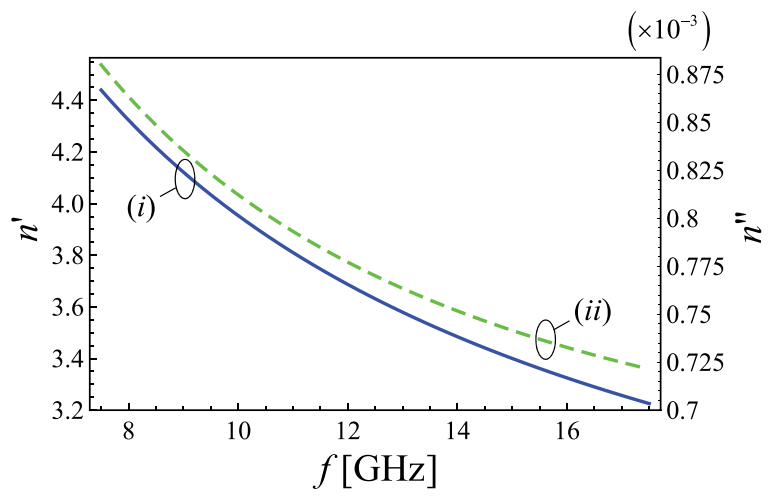

FIG. 1. Refractive index $\left(n=n^{\prime}+i n^{\prime \prime}\right)$ of the double wire metamaterial as a function of the frequency, calculated using Eq. (4) of Ref. 39. (i) Real part of the refractive index $\left(n^{\prime}\right)$ and (ii) imaginary part of the refractive index $\left(n^{\prime \prime}\right)$. The lattice constant $a=1.574 \mathrm{~mm}$, the radius of the wires is $r_{w}=0.05 a$, and the host dielectric is assumed to be RT/duroid $5880\left(\varepsilon_{\mathrm{h}}=2.2(1+i 0.00065)\right)$. The copper $(\mathrm{Cu})$ wires are modeled using a Drude dispersion model with parameters taken from the literature. ${ }^{40}$

infinitely large in the unbounded metamaterial. Thus, the refractive index of the effective medium is increasingly large for lower frequencies (Fig. 1), and in the limit of no loss, it diverges to $+\infty$ in the static limit. ${ }^{35}$

As already discussed in Ref. 35, a lossless anomalous dispersion regime is fully compatible with the KramersKronig relations for nonlocal media. Furthermore, it was shown in Ref. 39 that such a property may permit suppressing the chromatic aberrations inherent to conventional lenses, and that a compound lens formed by a thin glass layer coated with a thin double wire medium layer may be aberration free.

Here, we report an experimental verification of the reversal of the rainbow pattern by a metamaterial prism at microwaves. To this end, a prototype of a prism-shaped double wire metamaterial was fabricated using a layer-by-layer design and printed circuit board (PCB) techniques (Fig. 2). The metamaterial prism prototype is formed by a stack of 297 printed circuit boards RT/duroid 5880, with $\varepsilon_{\mathrm{h}}=2.2$, loss tangent $\delta=0.00065$, and thickness $a / 2=0.787 \mathrm{~mm}$. The circuit boards are parallel to the $x \mathrm{oz}$ plane. In this planar design, the original cylindrical metallic wires are replaced by printed metallic strips. Each circuit board was printed with parallel metallic strips with width $w_{s}=0.247 \mathrm{~mm}$. The metallic strips are tilted by $\pm 45^{\circ}$ with respect to the $z$-direction, such that the metallic strips in adjacent boards are mutually orthogonal. A horn antenna that operates in the $\mathrm{X}$ band is used to illuminate the oblique face of the metamaterial prism along the normal direction (Fig. 2). In order to measure the $x$-component of the electric field at the output plane (after propagation through the metamaterial prism), we used an electric field probe attached to the wrist of a moving robotic arm (shielded with microwave absorbing material-Eccosorb LS-26) and connected to a vector network analyzer (R\&S ZVB20).

The measured squared amplitude of the $x$-component of the electric field $\left|E_{x}\right|^{2}$ associated with the refracted beam is depicted in Fig. 3(a) for two different frequencies of operation: (i) $f=7.605 \mathrm{GHz}$ and (ii) $f=16.325 \mathrm{GHz}$. It is manifest from Fig. 3(a) that the transmission angle $\theta_{\mathrm{t}}$, defined by the direction normal to the output interface (z-direction) and (a)

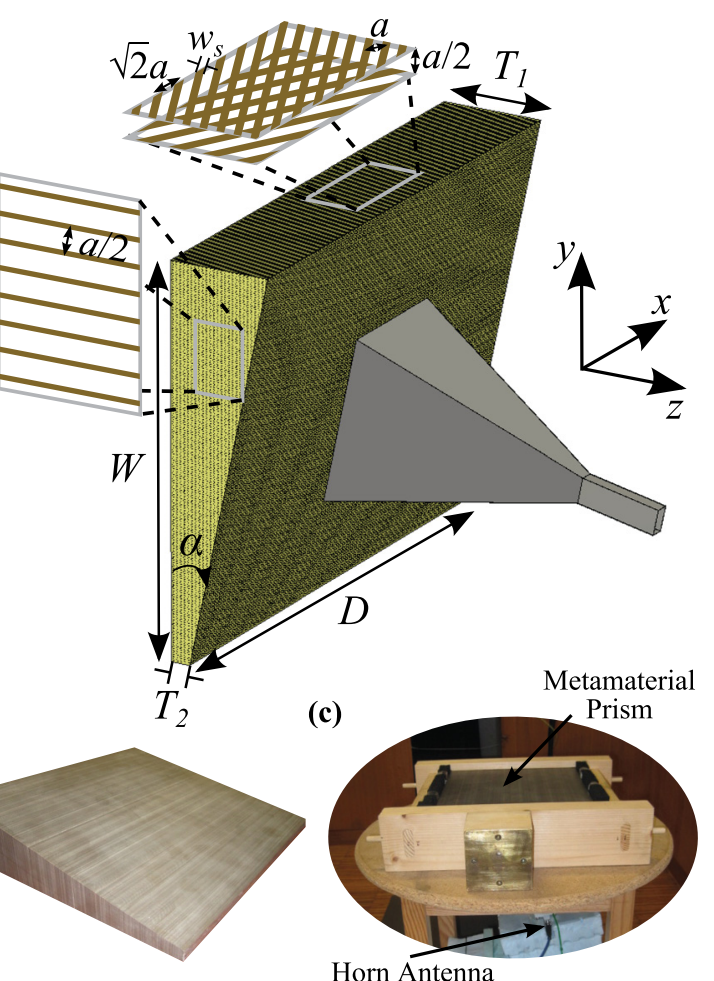

FIG. 2. Double wire metamaterial prism. (a) Geometry of the metamaterial prism: a stack of dielectric slabs is imprinted with an ultradense array of metallic strips with width $w_{s}$ and tilted by $\pm 45^{\circ}$ with respect to the $z$-direction. The metamaterial prism is excited by a horn antenna that illuminates the prism along the normal direction. The dimensions of the prism are $W=148 a=23.3 \mathrm{~cm}, D=28 \mathrm{~cm}, T_{1}=5.79 \mathrm{~cm}$, and $T_{2}=1.1 \mathrm{~cm}$. The prism apex angle $\alpha$ is $12^{\circ}$. (b) Photo of the fabricated metamaterial prism prototype. (c) Photo of the experimental setup.

the refracted beam, decreases with the frequency. According to Snell's law ( $\sin \theta_{\mathrm{t}}=n^{\prime} \sin \alpha$, where $n^{\prime}$ is the real part of the metamaterial refractive index $\left.n=\sqrt{\varepsilon}=n^{\prime}+i n^{\prime \prime}\right)$, if $\theta_{\mathrm{t}}$ decreases with frequency then the refractive index must decrease as well. Since the imaginary part of the metamaterial refractive index $n^{\prime \prime}$ is rather small compared to the real part $n^{\prime}$ (i.e., $n^{\prime} \gg n^{\prime \prime}$; see Fig. 1), this property can only be explained by the strong spatial dispersive response of the metamaterial. Thus, the experimental results of Fig. 3(a) support, indeed, that this wire-based metamaterial is characterized by a lowloss broadband anomalous material dispersion at microwaves.

We used a homemade spatial dispersive finite-difference frequency-domain (FDFD-SD) code ${ }^{41}$ to numerically simulate the electromagnetic response of the double wire metamaterial prism. This numerical approach models the metamaterial prism as a bulk material characterized by some effective parameters (for more details, see Ref. 41). In the FDFD-SD simulation, the metamaterial prism is infinitely extended along the $x$-direction, and finite along the $y$ - and $z$-directions. The metamaterial prism is illuminated along the normal direction by a Gaussian cylindrical beam characterized by a beam waist $2 w_{0}=96 \mathrm{~mm}$. These parameters were chosen so that the Gaussian beam mimics closely the field profile of the horn antenna excitation used in the experiment.

In Fig. 3(b), we show the FDFD-SD simulation results of $\left|E_{x}\right|^{2}$ in all regions of space for two different frequencies of operation: (i) $f=7.55 \mathrm{GHz}$ and (ii) $f=16.62 \mathrm{GHz}$. Clearly, these results are qualitatively consistent with the 
(a)

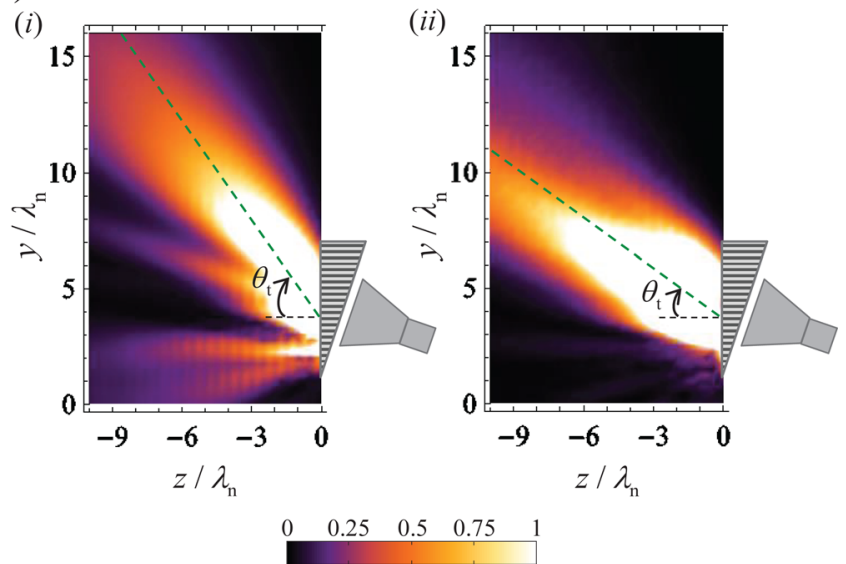

(b)
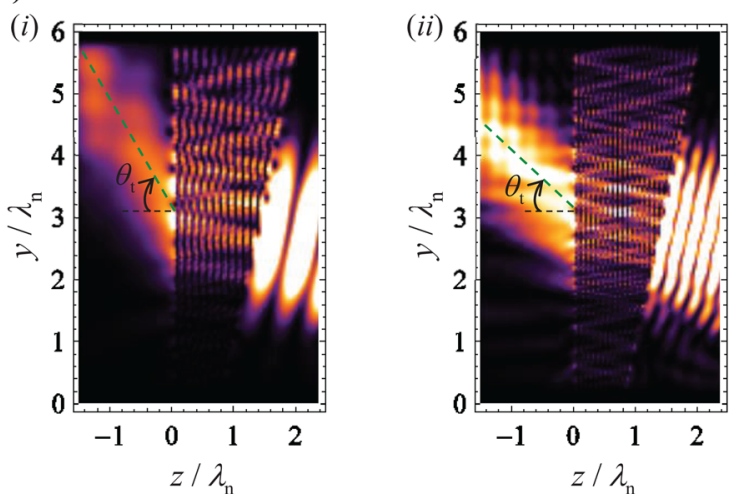

FIG. 3. Normalized squared amplitude of the electric field. (a) Density plot of the measured $\left|E_{x}\right|^{2}$ in the scanning plane $(z<0)$. (i) $f=7.605 \mathrm{GHz}$ and (ii) $f=16.325 \mathrm{GHz}$. To ease the interpretation of the results, a schematic drawing of the metamaterial prism and horn antenna (at full scale) is shown. The propagation is towards the left-hand side region. (b) Density plot of $\left|E_{x}\right|^{2}$ inside and outside the metamaterial prism calculated using a FDFD-SD simulation ${ }^{41}$ based on the effective medium model. (i) $f=7.55 \mathrm{GHz}$ and (ii) $f=16.62 \mathrm{GHz}$. The green dashed lines represent the refracted beam propagation direction, whereas the black dashed lines represent the direction normal to the interface. The spatial coordinates $y$ and $z$ are normalized to the reference wavelength $\lambda_{n}=39.71 \mathrm{~mm}$.

experimental results of Fig. 3(a), also showing that the transmission angle $\theta_{\mathrm{t}}$ decreases with the frequency.

We have also simulated the response of the metamaterial prism using the commercial electromagnetic simulator CST Microwave Studio. ${ }^{42}$ In this simulation, all the fine details of the microstructure of the metamaterial are taken into account. All the structural parameters are chosen in agreement with the experimental prototype, and similar to the experiment, a horn antenna is used to excite the structure. However, while in the experimental setup the horn antenna illuminates the oblique face of the prism, in the CST simulation the prism is illuminated through the opposite face to simplify the meshing of the computational domain.

The time snapshots $(t=0)$ of the $x$-component of the electric field obtained with CST Microwave Studio for two different frequencies of operation are depicted in Fig. 4. These results further validate the previously described experimental (Fig. 3(a)) and FDFD-SD numerical results (Fig. 3(b)), and further support that the double wire metamaterial prism is characterized by an anomalous dispersion regime.

In order to demonstrate the broadband nature of this property, we depict in Fig. 5 the transmission angle $\theta_{\mathrm{t}}$ as a (a)

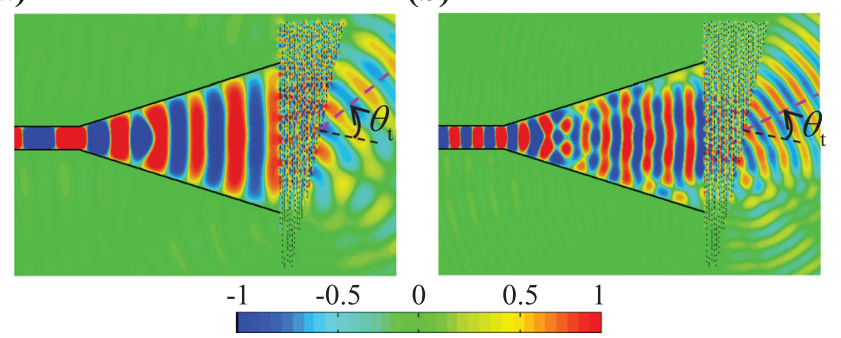

FIG. 4. Time snapshot of the normalized electric field $E_{x}(t=0)$ obtained from full-wave simulations done in CST Microwave Studio. ${ }^{42}$ (a) $f=8.9 \mathrm{GHz}$ and (b) $f=16 \mathrm{GHz}$. The purple dashed lines represent the refracted beam propagation direction, whereas the black dashed lines represent the normal to the interface.

function of the frequency in the $7.5-17.5 \mathrm{GHz}$ range, obtained with five different approaches: (i) the theoretical formula $\theta_{\mathrm{t}}=\arcsin \left(n^{\prime} \sin \alpha\right)$; (ii) a physical optics approximation similar to what is reported in Ref. 35; (iii) the FDFDSD method; ${ }^{41}$ (iv) the full-wave electromagnetic simulator; ${ }^{42}$ and (v) the experimental measurements. A least-squares fit was used to determine $\theta_{\mathrm{t}}$ for the (iii) FDFD-SD, (iv) CST, and (v) experimental results. Moreover, in order to check the accuracy of our least-squares based fitting method, we have also calculated the transmission angle using a spatial Fourier transform approach that allows us to determine the dominant $\left(k_{y}, k_{z}\right)$ wave vector components of an image and thereby $\theta_{\mathrm{t}}$. The maximum relative error between the transmission angles calculated with the two different fitting methods is about $5^{\circ}$.

As seen in Fig. 5, the qualitative agreement of the results obtained with the different approaches is very good. All the curves in Fig. 5 indicate that the transmission angle $\theta_{\mathrm{t}}$ decreases with frequency, contrarily to what happens in a conventional glass prism. On the other hand, one can see that the CST results are the ones that better match the experimental data. In CST simulation, we only obtained results for frequencies less than $16 \mathrm{GHz}$ due to limited computational resources. It may be noticed that all the other results are slightly above the experimental ones (by about $5^{\circ}$ ). This is simply related to the fact that with the exception of the CST

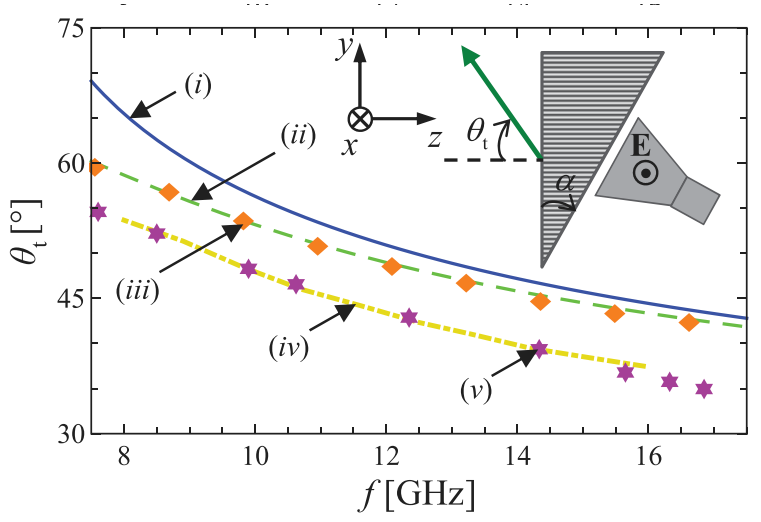

FIG. 5. Transmission angle $\theta_{\mathrm{t}}$ as a function of the frequency $f$. (i) Solid line: obtained using the theoretical formula $\theta_{\mathrm{t}}=\arcsin \left(n^{\prime} \sin \alpha\right)$; (ii) dashed line: obtained from the physical optics approximation; ${ }^{35}$ (iii) diamond-shaped symbols: calculated using a FDFD-SD simulator; ${ }^{41}$ (iv) dotted-dashed line: calculated using the full-wave electromagnetic simulator CST; ${ }^{42}$ and (v) star-shaped symbols: experimental results. 
simulation, all the other simulations assume a 2-dimensional setup wherein the prism and the source are invariant to translations along the $x$-direction.

In conclusion, we have experimentally verified that the transmission angle $\theta_{\mathrm{t}}$ of a beam refracted at the interfaces of a microwave metamaterial prism formed by nonconnected crossed metallic wires decreases with frequency in a wide spectral range. Our results provide a clear experimental proof of the low-loss broadband anomalous material dispersion regime predicted in Ref. 35. It is important to stress that, since the spatial dispersive response of the metamaterial persists even at high frequencies, and despite all the technological challenges, the reported phenomenon may be also experimentally demonstrated in the terahertz, infrared, or even optical frequency bands. ${ }^{39}$

This work was funded by Fundação para a Ciência e a Tecnologia under Project PTDC/EEI-TEL/2764/2012. T.A.M. acknowledges the financial support by Fundação para a Ciência e a Tecnologia (FCT/POPH) and the cofinancing of Fundo Social Europeu under the PostDoctoral fellowship SFRH/BPD/84467/2012.

${ }^{1}$ M. Born and M. Wolf, Principles of Optics, 7th ed. (expanded) (Cambridge University Press, 1999).

${ }^{2}$ L. D. Landau and E. M. Lifshitz, Electrodynamics of Continuous Media, 2nd ed. (Pergamon Press, Oxford, England, 1984).

${ }^{3}$ J. B. Pendry, Phys. Rev. Lett. 85, 3966 (2000).

${ }^{4}$ D. R. Smith, W. J. Padilla, D. C. Vier, S. C. Nemat-Nasser, and S. Schultz, Phys. Rev. Lett. 84, 4184 (2000).

${ }^{5}$ R. A. Shelby, D. R. Smith, and S. Schultz, Science 292, 77 (2001).

${ }^{6}$ N. Fang, H. Lee, C. Sun, and X. Zhang, Science 308, 534 (2005).

${ }^{7}$ D. Schurig, J. J. Mock, B. J. Justice, S. A. Cummer, J. B. Pendry, A. F. Starr, and D. R. Smith, Science 314, 977 (2006).

${ }^{8}$ J. Valentine, S. Zhang, T. Zentgraf, E. Ulin-Avila, D. A. Genov, G. Bartal, and X. Zhang, Nature 455, 376 (2008).

${ }^{9}$ P. A. Belov, Y. Hao, and S. Sudhakaran, Phys. Rev. B 73, 033108 (2006).

${ }^{10}$ G. Shvets, S. Trendafilov, J. B. Pendry, and A. Sarychev, Phys. Rev. Lett. 99, 053903 (2007).

${ }^{11}$ P. Ikonen, C. Simovski, S. Tretyakov, P. Belov, and Y. Hao, Appl. Phys. Lett. 91, 104102 (2007).

${ }^{12}$ P. A. Belov, Y. Zhao, S. Tse, P. Ikonen, M. G. Silveirinha, C. R. Simovski, S. Tretyakov, Y. Hao, and C. Parini, Phys. Rev. B 77, 193108 (2008).

${ }^{13}$ T. A. Morgado and M. G. Silveirinha, New J. Phys. 11, 083023 (2009).
${ }^{14}$ T. A. Morgado, J. S. Marcos, M. G. Silveirinha, and S. I. Maslovski, Appl. Phys. Lett. 97, 144102 (2010).

${ }^{15}$ M. G. Silveirinha and C. A. Fernandes, Phys. Rev. B 78, 033108 (2008).

${ }^{16}$ M. G. Silveirinha, C. A. Fernandes, J. R. Costa, and C. R. Medeiros, Appl. Phys. Lett. 93, 174103 (2008).

${ }^{17}$ T. A. Morgado, J. S. Marcos, M. G. Silveirinha, and S. I. Maslovski, Phys. Rev. Lett. 107, 063903 (2011).

${ }^{18}$ M. G. Silveirinha, C. A. Fernandes, and J. R. Costa, Phys. Rev. B 78, 195121 (2008).

${ }^{19}$ M. G. Silveirinha, C. R. Medeiros, C. A. Fernandes, and J. R. Costa, Phys. Rev. B 81, 033101 (2010).

${ }^{20}$ M. G. Silveirinha, Phys. Rev. B 79, 153109 (2009).

${ }^{21}$ T. A. Morgado, J. S. Marcos, S. I. Maslovski, and M. G. Silveirinha, Appl. Phys. Lett. 101, 021104 (2012).

${ }^{22}$ C. R. Simovski and P. A. Belov, Phys. Rev. E 70, 046616 (2004).

${ }^{23}$ I. S. Nefedov, A. J. Viitanen, and S. A. Tretyakov, Phys. Rev. B 72, 245113 (2005).

${ }^{24}$ P. A. Belov, R. Marqués, S. I. Maslovski, I. S. Nefedov, M. G. Silveirinha, C. R. Simovski, and S. A. Tretyakov, Phys. Rev. B 67, 113103 (2003).

${ }^{25}$ M. G. Silveirinha and C. A. Fernandes, IEEE Trans. Microwave Theory Tech. 53, 1418 (2005).

${ }^{26}$ C. R. Simovski, P. A. Belov, A. V. Atrashchenko, and Y. S. Kivshar, Adv. Mater. 24(31), 4229-4248 (2012).

${ }^{27}$ J. Yao, Z. Liu, Y. Liu, Y. Wang, C. Sun, G. Bartal, A. M. Stacy, and X. Zhang, Science 321, 930 (2008).

${ }^{28}$ F. Lemoult, M. Fink, and G. Lerosey, Nat. Commun. 3, 889 (2012).

${ }^{29}$ D. E. Fernandes, S. I. Maslovski, and M. G. Silveirinha, Phys. Rev. B 85, 155107 (2012).

${ }^{30}$ V. V. Vorobev and A. V. Tyukhtin, Phys. Rev. Lett. 108, 184801 (2012).

${ }^{31}$ A. N. Poddubny, P. A. Belov, and Y. S. Kivshar, Phys. Rev. A 84, 023807 (2011).

${ }^{32}$ A. Poddubny, I. Iorsh, P. Belov, and Y. Kivshar, Nat. Photonics 7, 948-957 (2013).

${ }^{33}$ I. S. Nefedov and C. R. Simovski, Phys. Rev. B 84, 195459 (2011).

${ }^{34}$ A. V. Kabashin, P. Evans, S. Pastkovsky, W. Hendren, G. A. Wurtz, R. Atkinson, R. Pollard, V. A. Podolskiy, and A. V. Zayats, Nat. Mater. 8, 867 (2009).

${ }^{35}$ M. G. Silveirinha, Phys. Rev. Lett. 102, 193903 (2009).

${ }^{36}$ S. Chu and S. Wong, Phys. Rev. Lett. 48, 738 (1982).

${ }^{37}$ M. D. Stenner, D. J. Gauthier, and M. A. Neifeld, Nature (London) 425, 695 (2003).

${ }^{38}$ J. P. Vigneron, P. Simonis, A. Aiello, A. Bay, D. M. Windsor, J.-F. Colomer, and M. Rassart, Phys. Rev. E 82, 021903 (2010).

${ }^{39}$ J. T. Costa and M. G. Silveirinha, Opt. Express 20, 13915 (2012).

${ }^{40}$ M. A. Ordal, R. J. Bell, R. W. Alexander, Jr., L. L. Long, and M. R. Querry, Appl. Opt. 24, 4493 (1985).

${ }^{41}$ J. T. Costa and M. G. Silveirinha, Phys. Rev. B 86, 075129 (2012).

${ }^{42}$ See http://www.cst.com, CST Microwave Studio ${ }^{\mathrm{TM}}$ 2014, CST GmbH for a MPI parallel computing solution was used to simulate this model in CST. 